\section{A influência da assistência profissional em saúde na escolha do tipo de parto: um olhar sócio antropológico na saúde suplementar brasileira}

\section{The influence of professional health care on the choice of type of childbirth: a socio- anthropological perspective on supplementary Brazilian health care}

\section{Abstract}

Objectives: this article reports on a multicentric research carried out in three State capitals in the Southern Region of Brazil, focusing on the experience of users of the supplementary network and aims to contribute to understanding of the influence of Professional care on the type of delivery carried out in this segment of the population.

Methods: a qualitative, exploratory and descriptive approach was adopted, using data collected between December 2006 and February 2007 by way of interviews with 33 women within 18 months of childbirth

Results: the results showed a high incidence of cesarean section and, among the factors contributing this was, identified the medicalization of the process of pregnancy and childbirth, the fear of pain, the convenience of a pre-established date of birth and a process of negotiation/orientation between the pregnant woman, her family and medical professionals, with the latter enjoying special authority. Cultural factors, previous experiences, family relations and pre-natal care based on biomedicine also inclined women in favor of cesarean birth.

Conclusions: it was concluded that a combination of cultural and organizational factors should be considered when restructuring pre-natal care with a view to promoting natural birth.

Key words Parturition, Cesarean section, Anthropology, Supplemental health, Health services
Denise Pires 1

Hosanna Pattrig Fertonani 2

Eleonor Minho Conill 3

Thaís Alves Matos 4

Fernanda Peixoto Cordova 5

Cíntia da Silva Mazur 6

1,4 Departamento de Enfermagem. Universidade Federal de Santa Catarina. Campus Universitário, S/N. Florianópolis, SC, Brasil. CEP: 88.040-970. E-mail: piresdp@yahoo.com

2 Departamento de Enfermagem. Universidade Estadual de Maringá. Maringá, PR, Brasil.

3 Departamento de Saúde Pública. Universidade Federal de Santa Catarina. Florianópolis, SC, Brasil.

5 Centro Universitário Metodista do Sul, IPA, RS. Porto Alegre, RS, Brasil.

6 Escola de Saúde das Faculdades Integradas do Brasil (UNIBRASIL)- PR. Curitiba, PR, Brasil.

\section{Resumo}

Objetivos: este estudo é um recorte de uma pesquisa multicêntrica realizada em três capitais da Região Sul do Brasil que focaliza as experiencias de usuárias da rede suplementar, buscando contribuir na compreensão da influência da assistência profissional na escolha do tipo de parto realizado neste segmento.

Métodos: estudo de abordagem qualitativa, exploratório-descritivo, utilizando dados coletados entre dezembro de 2006 e fevereiro de 2007, através de entrevistas com 33 mulheres com até dezoito meses de pós-parto.

Resultados: os resultados mostraram alta incidência de parto cesáreo e entre os fatores que contribuiram para este fenômeno identificou-se a medicalização do processo gravidez-parto, o medo da dor, a conveniência da data marcada e um processo de negociação/orientação que se estabelece entre a gestante e seus familiares e o profissional médico, fortemente influenciado pelo vínculo de confiança dessa relação. Fatores culturais, experiências anteriores, relações familiares e a assistência pré-natal baseada na biomedicina também influenciaram a favor do parto cesáreo.

Conclusões: conclui-se que um conjunto de fatores de ordem cultural e organizacional deva ser considerado no sentido da reorganização da assistência pré-natal, visando à implantação de práticas que favoreçam o parto normal.

Palavras-chave Parto, Cesárea, Antropologia, Saúde Suplementar, Serviços de Saúde 


\section{Introdução}

Estima-se que o subsetor da saúde suplementar seja responsável pela cobertura de $25 \%$ da população brasileira, o que na Região Sul corresponde a 43\%, $46 \%$ e $37 \%$ da população de Curitiba, de Florianópolis e de Porto Alegre, respectivamente. Estudos da Agência Nacional de Saúde Suplementar (ANS) acerca da regulação e mais recentemente relacionados às características do modelo assistencial e da qualidade dos serviços prestados por este segmento indicam que a assistência na situação do parto é marcada pela fragmentação e pela exacerbada utilização de procedimentos biologicistas. ${ }^{1}$

Analisando o Sistema de Informação sobre Nascidos Vivos (Sinasc) do Ministério da Saúde (MS), as cesáreas representaram em 2004 mais de $41 \%$ dos partos realizados no país, sendo que a Organização Mundial da Saúde (OMS) aconselha que esse percentual não ultrapasse $15 \% .2,3$ Esse elevado índice de partos cesáreos no contexto brasileiro se destaca como alarmante, principalmente ao comparar-se com dados de países como Holanda (14\%) e Estados Unidos (26\%). ${ }^{4}$

Tal situação é mais grave entre as usuárias de planos de saúde, pois o percentual de partos cesáreos vem crescendo, correspondendo a $64,3 \%$ do total de partos em 2003, e 80,7\% em 2006.3 Esses dados sinalizam uma preocupante importância epidemiológica, pois representam significativo impacto nos serviços e na saúde das mulheres submetidas a tal procedimento. 5

Para a compreensão do referido fenômeno é importante que se considere a sua múltipla determinação, que inclui a organização dos serviços de saúde e a cobertura da assistência pré-natal, as relações entre os temas gênero e saúde, 6,7 e a existência, na sociedade atual, de uma pluralidade de sistemas de cuidados que ultrapassam os limites da rede institucionalizada de serviços de saúde.

O referencial da socio-antropologia, que analisa o fenômeno saúde-doença-cuidados como parte de macrocontextos, determinados econômica e socialmente, incorporando a dimensão subjetiva e cultural, é útil para o estudo dessa temática porque possibilita a apreensão da dimensão macrossocial e também das diferentes percepções, interpretações e comportamentos de profissionais e usuários. ${ }^{8-12}$ Como categoria analítica, a experiência de adoecer (ou vivenciar situações que demandam cuidados em saúde) se refere à forma como os indivíduos e grupos sociais assumem essa situação, o que inclui os meios utilizados para responder às suas necessidades.

Apesar da indiscutível importância dessa cate- goria, estudá-la implica enfrentar um dos grandes desafios da socio-antropologia contemporânea, ou seja, a compreensão das dimensões cognitivas e sociais que são incorporadas e se expressam nas representações individuais. A experiência de doença desvela aspectos tanto sociais como cognitivos, tanto subjetivos (individuais) como objetivos (coletivos), importando conhecer o movimento pelo qual as práxis individuais e a generalidade do social se constituem e reconstituem mutuamente. 13

As práticas em saúde envolvem relações entre seres humanos que vivenciam papéis diferenciados: o de terapeuta e o de pessoa portadora de alguma carência que necessita de cuidado em saúde. Nesse encontro fatores de ordem cultural, social e de gênero, assim como as diferentes crenças acerca da vida, da saúde, da doença e do tratamento pertinente, se expressam e influenciam as escolhas de profissionais e usuários, assim como os resultados do processo. $7-10,13$

Durante a gravidez e o parto, as mulheres vivenciam várias alterações físicas e emocionais e expressam, neste processo, valores e crenças, assim como se defrontam com a estrutura social e cultural dos profissionais dos serviços de saúde. Ao procurarem os serviços para atendimento das necessidades durante a gravidez e parto, trazem consigo expectativas e preocupações que têm relação com as experiências de vida delas. Os profissionais, durante o exercício da prática assistencial, também expressam sua maneira de compreender a doença, influenciada por aspectos culturais, sociais e pelo modelo de formação hegemônico. $\mathrm{O}$ encontro entre esses dois sujeitos é sempre singular e influenciado pela dimensão cultural e social; e, embora seja um espaço propício ao diálogo, é permeado por uma relação assimétrica de poder e de saber com grande potencial de influência na escolha do tipo de parto, do itinerário terapêutico e da qualidade da assistência prestada às gestantes. ${ }^{8-10}$

Além disso, na assistência à mulher se expressam visões histórico-sociais e culturais acerca do feminino, do corpo da mulher, assim como os limites de sua autonomia para tomar decisões no processo de parturição. Na sociedade atual, apesar das conquistas do movimento feminista a partir da segunda metade do século passado, ainda é significativa a medicalização do fenômeno gravidez-parto. A medicalização do corpo feminino no século XIX ocorre em um contexto de exaltação da maternidade, transformando-a em objeto da medicina e deslocando o papel de partejar das parteiras para os médicos. 6

Então, sob a legitimação da ciência e da biome- 
dicina, institucionaliza-se a assistência pré-natal e o parto, o qual passou a ser percebido, basicamente, como um evento biológico, enfatizando-se os procedimentos técnicos "sobre o aparelho reprodutor" feminino em detrimento da percepção da complexidade da mulher em sua individualidade e inserção sócio-cultural. Tal processo foi acompanhado de perda de autonomia pelas mulheres e aumento da autoridade dos médicos para tomar decisões sobre o corpo feminino e sobre o parto. ${ }^{7}$

A escolha da mulher pelo parto normal ou cesáreo sofre, portanto, diversas influências. Entre elas destacam-se: os significados do papel da mulher nos diversos cenários sócio-culturais; o processo histórico de medicalização do corpo feminino; a compreensão familiar a respeito da gravidez, considerando-se que neste processo há uma expectativa que envolve toda a família, o parceiro e outros membros da relação social da mulher; suas experiências e vivências anteriores e, também, o paradigma que informa o modo como é prestada a assistência em saúde e sua organização.6-7,10 Nesse sentido, o presente estudo tem por objetivo contribuir para a compreensão da influência da assistência profissional em saúde na escolha do tipo de parto de gestantes atendidas pelos serviços de saúde suplementar.

\section{Métodos}

Trata-se de um estudo sob abordagem qualitativa, exploratório-descritivo, acerca das experiências referidas por usuárias dos serviços da saúde suplementar, durante a vivência do processo gravidezparto, em três capitais da Região Sul do Brasil. Consiste no recorte de uma pesquisa multicêntrica que investigou a relação público-privado, e os arranjos assistenciais nos itinerários terapêuticos de usuários/as da saúde suplementar em quatro linhas de cuidado e situações marcadoras, entre elas a materno-infantil, com o marcador "parto". 1

A amostra total foi composta por 33 mulheres que haviam dado a luz há até um ano e meio antes da data da coleta de dados (dezembro de 2006 a março de 2007) e que tinham cobertura de algum tipo de plano ou seguro de saúde. Para a seleção da população de mulheres do estudo recorreu-se a dois tipos de processos de composição de amostra: intencional e por conveniência. Em Florianópolis selecionou-se por meio da listagem dos nascimentos ocorridos em 2006, fornecida pela Secretaria Municipal de Saúde e obtida pelos registros das maternidades; em Curitiba a seleção ocorreu por convite para participar da pesquisa durante consultas de puericultura em um centro de atendimento infantil e em Porto Alegre foram selecionadas as usuárias de planos e seguros de saúde que acessavam unidades básicas de saúde para realização de imunização e teste do pezinho em seus filhos.

Os dados coletados através de entrevistas semiestruturadas respeitaram o disposto na Resolução 196/96 do Conselho Nacional de Saúde (CNS), incluindo a obtenção do consentimento em participar da pesquisa e aprovação no Comitê de Ética em Pesquisa do Instituto de Cardiologia da Secretaria de Estado da Saúde de Santa Catarina.

Com vistas à preservação do anonimato, as falas das entrevistadas foram identificadas por códigos, conforme a cidade onde foi realizada a entrevista $(\mathrm{F}=$ Florianópolis, $\mathrm{C}=$ Curitiba, $\mathrm{P}=$ Porto Alegre $)$, seguidos de números, de acordo com a ordem de entrevista por município.

A análise dos dados foi realizada à luz da análise temática de Bardin, 14 iniciando com a leitura flutuante de cada entrevista para uma visão do conjunto e uma imersão na narrativa das entrevistadas. A seguir foi feita uma leitura transversal para validar as précategorias analíticas, construídas no processo de elaboração do roteiro de entrevistas. Tal processo de análise foi facilitado com a utilização do software Qualitative Solutions Research NVivo (QSR NVivo) 2.0.15 Após codificação e análise dos dados obtidos emergiram duas categorias finais: a) expectativas em relação à gravidez e ao parto; b) fatores que interferem na decisão pelo tipo de parto e influência da assistência profissional durante o período de gestação para a escolha do tipo de parto.

\section{Resultados}

$\mathrm{Na}$ análise da população estudada, verificou-se uma correspondência entre condições econômicas e cobertura de plano privado, sendo que $84,8 \%$ das entrevistadas declararam renda entre 05 e 22 salários mínimos; a idade variou entre 20 e 40 anos, com predomínio entre 30 e 40 anos; a maioria das entrevistadas tinha um bom nível de escolaridade (pelo menos ensino médio incompleto, sendo que $30 \%$ concluíram o ensino superior e $18 \%$ a pós-graduação). No que diz respeito ao tipo de parto, observou-se alta incidência de parto cesáreo $(81,8 \%$ das entrevistadas) e com relação à operadora, predominou a modalidade de cooperativa médica $(63,6 \%)$, o que corresponde à realidade da Região Sul.

Quanto às expectativas em relação à gravidez $\mathrm{e}$ ao parto destacaram-se nas entrevistas sentimentos de satisfação com o fato de estar grávida, mas 
também de medo e ansiedade em relação ao parto:

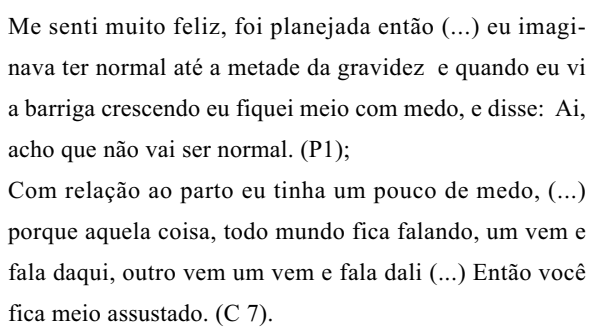

Os resultados mostraram que a maioria das mulheres manifestou o desejo de realizar o parto normal após a confirmação da gravidez, porém, à medida que realizavam as consultas de pré-natal ou se aproximava o momento do parto, esse desejo era substituído pelo do parto cesáreo. Algumas vezes a decisão pelo tipo de parto ocorreu no momento do parto:

Eu queria que fosse normal, mas como do primeiro eu tentei parto normal e não consegui, e acabei fazendo uma cesárea de emergência, então a médica indicou a cesárea daí eu já marquei a data, já resolvi. Eu queria que de repente antes da data marcada ele resolvesse nascer, mas isso não aconteceu então aí foi cesárea mesmo. (P10)

Os relatos das entrevistadas permitiram identificar alguns dos fatores que contribuem para a decisão a favor do parto cesáreo e que dizem respeito, de um lado, aos aspectos culturais e experiências anteriores das gestantes e de seus familiares e de outro, às crenças dos profissionais e a relação estabelecida durante a assistência pré-natal.

Entre esses fatores, destacam-se as questões relacionadas à mulher, como medo da dor, a conveniência da data marcada para organização do cotidiano familiar e a opção por tal tipo de parto:

Eu queria cesárea mesmo. Fiquei com medo de fazer parto normal, fiquei com medo de não fazer a coisa certa na hora certa (...) Eu ouvia até queria ter coragem, eu não tive coragem mesmo, eu queria ter coragem de fazer parto normal. Eu ouvia dizer que às vezes a mãe demorava muito e a criança tomava água e ficava, sabe? Até engolia o cocô dela mesmo, sabe essas coisas assim?! E eu ficava apavorada, daí não, quero cesárea mesmo, por não ter coragem. (F94);

A gravidez não foi planejada, mas o parto sim (...) fiz a cesariana quando estava com umas 38 semanas, 39 semanas prá não ter perigo de passar (...) também por causa das férias do meu marido, prá ele poder estar comigo, prá poder ter um apoio, porque fica complicado sem esse apoio, então a gente conseguiu conciliar tudo. (P4)
No que se refere às questões relacionadas ao profissional que acompanha o pré-natal e o parto, nota-se que a qualidade da orientação recebida pela gestante, ou até mesmo a falta de informação a respeito do parto normal, também interferem na decisão da mulher e da família desta, fazendo-as optar pelo parto cesáreo:

Até o parto não recebi muitas orientações, pra mim foi bem complicado até. Pra eu ter nenê não sabia como ia ser (...) o médico não queria muito conversar sobre o parto, eu tava em dúvida entre parto normal e a cesárea né? Depois eu fiquei sabendo que parto normal hoje tem, (...) como é que se diz? Menos dor e eu nem fui informada, fiquei sabendo até agora com essa novela que tá passando. (C6)

Destaca-se aqui que tanto para a realização de cesárea quanto para o parto normal a argumentação técnica tem um peso significativo na decisão da mulher e, durante o pré-natal, o médico teria um espaço importante para influenciar positivamente a favor do parto normal. No entanto, parece que a influência a favor da cesárea tem sido mais convincente. Muitas vezes a cesárea é incentivada sem uma indicação clara, como podemos perceber na fala de algumas entrevistadas.

Antes de saber que tava grávida eu queria cesárea, quando fiquei grávida comecei a me informar e quis parto normal, daí não foi possível, porque meu filho tinha $4 \mathrm{Kg}$ no último ultrassom aí o médico não fazia, quis optar pela cesárea. (F7);

Eu queria que fosse cesárea, porque lá em São Paulo o meu médico incentivava a cesárea, não é que ele incentivava, ele achava que era melhor, tanto prá criança quanto prá mim. Mais prá criança até do que pra mim. (F5)

Contudo, mesmo neste contexto de ênfase da cesariana, identificou-se, ainda que minoritária, a interferência do obstetra a favor do parto normal, em um processo de negociação entre as opiniões e expectativas da gestante e o posicionamento do médico, mesmo que a posição a favor da cesárea tenha predominado.

Então foi assim, durante a gravidez na primeira hora que eu tive certeza que tava grávida, eu assim: "vou fazer cesárea", decisão minha, né?! Depois o médico me convenceu do contrário, e depois eu convenci ele de que eu queria fazer cesárea $(\mathrm{F} 1)$.

A assistência no pré-natal, parto e puerpério, no segmento da saúde suplementar, é, predominante- 
mente, sinônimo de assistência médica e da lógica de cuidados da biomedicina, ou seja, de avaliação clínico-laboratorial e monitoramento no processo de parturição. No contexto de preparação para o parto, do total de entrevistadas uma minoria mencionou ter buscado assistência de outros profissionais de áreas como enfermagem, fisioterapia e psicologia, ou outras atividades de cuidado em saúde que considerassem a perspectiva da integralidade da assistência.

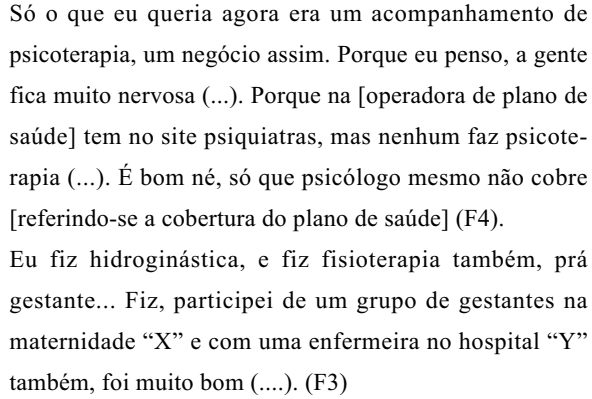

\section{Discussão}

Os dados obtidos aproximam-se do encontrado na literatura da antropologia aplicada à saúde.8-13,16 Estes estudos mostram que a percepção sobre a saúde, a vivência da doença e de intercorrências ou situações especiais, como é o caso do parto, envolvem uma explicação pessoal fortemente influenciada pela cultura, que interfere nas escolhas e no itinerário percorrido em busca de cuidados. Por outro lado, a literatura também registra a influência dos serviços de saúde, em especial das crenças e conhecimentos dos profissionais médicos, no curso dos cuidados durante o período grávido-puerperal e na escolha do tipo de parto. ${ }^{10}$

O período que antecede o parto é caracterizado por incertezas, pelo enfrentamento de situações desconhecidas, como o acesso à instituição, o desconhecimento da sua dinâmica e de como será prestada a assistência, provocando sentimentos de insegurança e estresse ${ }^{17}$ que poderão influenciar também na escolha do tipo de parto.

É importante considerar que durante a prática assistencial, como menciona Boltanski, 18 especialmente diante do médico, ocorre um colocar-se em frente ao desconhecido, o que gera ansiedade e tensões "porque o objeto que o sujeito abandona às manipulações do especialista e ao seu discurso, não é nada mais do que, no caso, o seu próprio corpo".
$\mathrm{Na}$ análise dos dados, pode-se observar que as decisões sobre o tipo de parto foram influenciadas pelos profissionais médicos, mas também, pelas expectativas das gestantes, em especial pelo medo da dor e riscos que acreditavam estar envolvidos no parto natural, assim como pelas suas experiências anteriores. O parto cesáreo parece ser compreendido pelas mulheres como indolor, mais bem assistido e seguro, além de permitir uma melhor organização do cotidiano da sua vida familiar. Tal entendimento por parte das entrevistadas possivelmente estaria relacionado a uma falta de preparação dessas para o parto normal durante as consultas de pré-natal, espaço este privilegiado para a discussão das questões relacionadas às principais diferenças, vantagens e desvantagens dos tipos de parto, assim como para a promoção da autonomia da mulher nessa escolha. 19,20

O encontro entre gestante e profissional de saúde é marcado pela influência da histórica exaltação e hegemonia do profissional médico como responsável por ordenar e normalizar questões referentes ao corpo feminino.7,21 Os dados da pesquisa sugerem que os altos índices de cesárea encontrados entre as mulheres assistidas neste segmento são influenciadas pelo poder médico e pelo paradigma da biomedicina. Mesmo em um grupo de mulheres de privilegiado nível socioeconômico e educacional como o estudado, predominou, majoritariamente, a decisão médica pelo parto cesáreo, ainda que a maioria delas inicialmente desejasse o parto normal.

Para o Ministério da Saúde (MS) e a Organização Mundial da Saúde (OMS) o preparo para o parto deve acontecer desde o pré-natal, onde se procura garantir à mulher o direito de escolha, mas contribuindo para o seu empoderamento com vistas ao parto normal. ${ }^{20}$ Ao mencionarem os sentimentos e expectativas em relação ao tipo de parto, as entrevistadas relataram medo da anestesia e da dor. Em 2004, Rugolo22 também encontrou resultados semelhantes em estudo sobre sentimentos e percepções, realizado com mães adolescentes, o qual mostra a necessidade da valorização do aspecto emocional na assistência à gestante, no nascimento $\mathrm{e}$ nos cuidados ao recém-nascido.

O predomínio do modelo assistencial da biomedicina favorece o olhar para o processo grávidopuerperal como um fenômeno biotécnico e não como uma vivência pessoal e sócio-cultural. Mas esse processo é permeado por uma contradição central, pois seu resultado leva a uma intervenção que se caracteriza, muitas vezes, por uma inadequação 
técnica do cuidado. Em estudo realizado por Cordova, ${ }^{19}$ com mulheres usuárias do subsetor de saúde suplementar, esse predomínio aparece como uma desvalorização da associação entre parto e fisiologia materna, pois os profissionais tendem a orientar o pré-natal por meio de uma lógica invertida: ao invés de prepararem as mulheres para o natural que seria o parto normal, a preparação e a escolha ocorre na direção do parto cirúrgico, tecnicamente reservado para as intercorrências.

Apesar de algumas medidas recentes da Agência Nacional de Saúde na direção de uma maior integralidade da atenção, a limitada cobertura de outras práticas profissionais além da assistência médica, por parte dos planos de saúde, colabora para a centralidade da influência da decisão médica na escolha final das mulheres. Além disso, as expectativas relacionadas ao parto são influenciadas pela divulgação de efeitos benéficos e protetores dos avanços tecnológicos, especialmente presentes em hospitais e laboratórios, o que favorece a identificação do ambiente hospitalar como seguro para a assistência ao parto. Faisal-Cury e Menezes23 apontam que a postura do profissional no acompanhamento pré-natal, ao não abrir espaço para o diálogo sobre as vias de parto, pode resultar numa avaliação superestimada da segurança do parto cirúrgico, o que também foi observado nesta pesquisa. Por outro lado, apesar do forte predomínio da indução ao parto cirúrgico, os resultados registraram, também, a existência de um processo de diálogo e negociação entre o profissional médico e a gestante em defesa do parto normal, demonstrando que o fenômeno é multifacetado.

$\mathrm{Na}$ assistência ao pré-natal e ao parto é importante considerar a complexidade do fenômeno, o papel dos diversos profissionais de saúde e o conjunto das necessidades das gestantes. ${ }^{17,19} \mathrm{O} \mathrm{MS}$, a OMS e a ANS reconhecem como indispensável a realização de mudanças no paradigma excessivamente medicalizado da assistência obstétrica como estratégia para reduzir as elevadas taxas de cesárea e recomendam a adoção de medidas em direção a integralidade e humanização do cuidado.4,20,24,25

\section{Considerações finais}

Os resultados da pesquisa mostraram que, mesmo em um grupo de mulheres com melhor renda e escolaridade, no qual seria esperado um maior poder de decisão sobre o próprio corpo, influenciando positivamente no sentido do parto natural, verifica-se altíssima incidência de cesáreas. A abordagem qualitativa, com um referencial sócio-antropológico, mostrou-se útil para ampliar a compreensão acerca da escolha do tipo de parto ao identificar a importância da autoridade médica e das representações de maior segurança e menor sofrimento físico relacionadas com o parto cesáreo.

Os dados revelaram que há falta de informações adequadas a favor do parto normal durante o prénatal, realizado quase que exclusivamente pelo profissional médico, o que nos leva a concluir que mudanças neste processo de diálogo e negociação poderão contribuir para diminuir a alta incidência de parto cesáreo. No entanto, a complexidade do fenômeno indica a necessidade de ampliar o processo de investigação, inclusive comparando as taxas de cesárea em diferentes modelos de atenção.

\section{Agradecimentos}

À Agência Nacional de Saúde Suplementar pelo financiamento da pesquisa; e ao Dr. Ricardo Burg Ceccin pela Coordenação do Núcleo Sul da Rede de Centros Colaboradores em Saúde Suplementar, responsável pelo projeto regional que inclui o subprojeto "Relação público-privado e arranjos tecno-assistenciais na utilização de serviços de saúde: um estudo de itinerários terapêuticos na região sul”.

\section{Referências}

1. Conill EM, Pires D, Sisson MC, Oliveira, MC, Boing AF, Fertonani HP. O mix público privado na utilização de serviços de saúde: um estudo dos itinerários terapêuticos de beneficiários do segmento de saúde suplementar brasileiro. Ciênc Saúde Coletiva. 2008; 13: 1501-10.

2. Brasil. Ministério da Saúde. DATASUS. Indicadores e dados básicos. 2005. [acesso em 2009 Fev 28] Disponível em: http://www.datasus.gov.br.
3. Brasil. Agência Nacional de Saúde Suplementar. O modelo de atenção obstétrica no setor de Saúde Suplementar no Brasil: cenários e perspectivas. 2008. [acesso em 2008 Out 01] Disponível em: http://bvsms.saude.gov.br/bvs/publicacoes/modelo_atencao_obstetrica.pdf

4. Brasil. Agência Nacional de Saúde Suplementar. A epidemia do parto cesáreo. 2008. [acesso em 2008 Out 01] Disponível em: http://www.ans.gov.br/portal/upload/noticias/_Hotsite_Parto/20080201.pdf. 
5. Coimbra LC. Fatores associados à inadequação da assistência pré-natal. Rev Saúde Pública. 2003; 37: 456-62.

6. Martins APV. Visões do feminino: a medicina da mulher nos séculos XIX e XX. Rio de Janeiro: FIOCRUZ; 2004.

7. Vieira EM. A medicalização do corpo feminino. $1^{\mathrm{a}}$ reimp. Rio de Janeiro: FIOCRUZ; 2008.

8. Kleinman A. Patients and healers in the context of culture an exploration of the borderland between, antropology, medicine and psychiatric. London: University of Califórina 1980 .

9. Langdon EJ. A doença como experiência: o papel da narrativa na construção sociocultural da doença. Etnográfica. 2001; 5: 241-60.

10. Helman CG. Cultura, saúde e doença. 4. ed. Porto Alegre: Artes Médicas; 2003.

11. Minayo MCS. Contribuições da antropologia para pensar a saúde In: Campos GVS, Minayo MCS, Akerman M, Drumond J, Carvalho YM. orgs. Tratado de Saúde Coletiva. São Paulo/Rio de Janeiro: HUCITEC/FIOCRUZ; 2006. p. 201-30.

12. Silva DMGV, Meirelles BHS, Souza SS, Francioni F. O itinerário terapêutico de pessoas com problemas respiratórios crônicos. Texto Contexto-Enferm. 2004; 13: 50-6.

13. Alves PC. A experiência da enfermidade: considerações teóricas. Cad Saúde Pública. 1993; 9: 263-71.

14. Bardin L. Análise de conteúdo. Lisboa: Edições 70; 1995.

15. Guizzo BS, Krziminski CO, Oliveira DLLC. O software QSR NVivo 2.0 na análise qualitativa de dados: ferramenta para a pesquisa em ciências humanas e da saúde. Rev Gaúch Enferm. 2003; 24: 53-60.

16. Alves PC, Souza IM. Escolha e Avaliação de Tratamento para Problemas de Saúde: considerações sobre o itinerário terapêutico. In: Rabelo MCM, Alves PC, Souza IMA. orgs. Experiência de doença e narrativa. Rio de Janeiro: FIOCRUZ; 2004. p. 125-38.
17. Melleiro MM, Gualda DMR. Experiências e expressões de gestantes na interação com o sistema de saúde: um enfoque fotoetnográfico. Rev Latino-Am Enferm. 2004; 12: 503-10.

18. Boltanski L. As classes sociais e o corpo. Rio de Janeiro: Graal; 1979.

19. Cordova FP. Do pré-natal ao parto: estudo das trajetórias terapêuticas percorridas por um grupo de mulheres usuárias do subsetor suplementar de assistência à saúde [dissertação]. Porto Alegre: Programa de Pós-Graduação em Enfermagem da Universidade Federal do Rio Grande do Sul; 2008.

20. Brasil. Ministério da Saúde. Pré-natal e puerpério: atenção qualificada e humanizada. Brasília, DF; 2005.

21. Costa T, Stotz EM, Grynszpan D, Souza MCB. Naturalização e medicalização do corpo feminino: o controle social por meio da reprodução. Interface. $2006 ; 10$ : 363-80.

22. Rugolo LM. Sentimentos e percepções de puérperas com relação à assistência prestada pelo serviço materno-infantil de um hospital universitário. Rev Bras Saúde Matern Infant. 2004; 4: 423-43.

23. Faisal-Cury AF, Menezes PR. Fatores associados à preferência por cesareana. Rev Saúde Pública. 2006; 40: 226-32.

24. Brasil. Ministério da Saúde. Área Técnica de Saúde da Mulher. Parto, aborto e puerpério - assistência humanizada à mulher. Brasília, DF; 2001.

25. Brasil. Ministério da Saúde. Agência Nacional de Saúde Suplementar. Resolução Normativa $n^{\circ} .167$ de 9 de janeiro de 2007. Rio de Janeiro; 2007.

Recebido em 14 de outubro de 2008

Versão final apresentada em 26 de janeiro de 2010

Aprovado em 18 de fevereiro de 2010 